\title{
LETTER THE THE EDITOR
}

\section{SHOULD SCHOOL-AGE CHILDREN NOT ATTENDING SCHOOL AND ADULTS AGED 20-44 YEARS BE CONSIDERED HIGH-RISK GROUP FOR BACILLARY DYSENTERY INFECTION IN GUANGZHOU, CHINA? A REVIEW OF 4775 BACILLARY DYSENTERY CASES}

January 13, 2014

\section{To the Editor:}

Bacillary dysentery (BD), also known as Shigellosis, is the most common intestinal infectious disease caused by Shigella, characterized by the passage of loose stools mixed with blood and mucus and accompanied by fever, abdominal cramps and tenesmus. Organisms as low as 10-00 in number can cause the disease ${ }^{10}$. The infection can be transmitted by the fecal-oral route via contaminated water, food, or person-to-person contact $^{6}$.

Worldwide, the incidence of BD is estimated to be 164.7 million cases per year, of which 163.2 million were in developing countries, where 1.1 million deaths occurred ${ }^{12}$. In Guangzhou, the largest trading city in southern China, BD is still one of the most common intestinal infectious diseases. It was reported that during the period of 1997-2005, the annual incidence rate of BD in Guangzhou ranged from 15.24 to 20.50 per $100,000^{15}$, leading public health authorities to be concerned about its high incidence. To address this issue, in 2006, the Guangzhou municipal government launched an Intestinal Infectious Disease Surveillance, Prevention and Control Program (IIDSPCP). According to IIDSPCP, once a patient was diagnosed with suspected or confirmed $\mathrm{BD}$, a series of preventive measures such as avoiding direct contact with infective patients, disinfection of virally contaminated items or premises, and good personal hygiene practices have been strongly recommended and sometimes mandatorily implemented by local government.

In this study, we obtained data of BD cases in Guangzhou during the period of 2006-2012, from the National Notifiable Disease Report System (NNDRS). In China, BD is a notifiable Class-B communicable disease. This means, as for other national reportable diseases, the BD cases are diagnosed according to the unified diagnostic criteria issued by the Chinese Ministry of Health, for a patient's illness to meet the case definition for BD the clinical signs (diarrhea, fever, abdominal pains, tenesmus, and stool mixed with blood, etc.) must be present and samples should be taken for laboratory confirmation (defined as 1), identification of Shigella from stools by culture or 2) and detection of Shigella genetic material from stools by PCR. A standard form was used by local physicians and epidemiologists to collect individual information on each BD case, including age, address, date of onset, diagnosis, and laboratory test result, etc. Routine case reporting is done by hospitals through NNDRS within 24 hours after diagnosis. Descriptive statistics are used to describe the basic features of BD confirmed cases in the study, and chi-square test was used to examine linear trends in proportions by years. Analyses were carried out using SAS (V.8.01, SAS Institute, Cary, New Jersey, USA).

From January $1^{\text {st }}, 2006$ to December $31^{\text {st }}, 2012$, a total of 4775 BD confirmed cases were reported in Guangzhou, of which $55.4 \%$ (2481) were male patients and $44.6 \%$ (1994) were female patients. Annual incidence rates from 2006 to 2012 were 12.43, 9.96, 6.42, 4.44, 3.21, 2.35, and 1.84 (per 100000 ), respectively (Fig. 1). Ages ranged from two months to 101 years old (mean age was 28.6 years). The proportion of confirmed cases between $<5,6-19,20-44,45-64$, and $>65$ was $36.08 \%$ (1723), 7.48\% (357), 35.48\% (1694), 13.24\% (632), and 7.73\% (369), respectively. Of particular note, the proportion of patients identified as school-age children (6-15 years) not attending school from 2006 to 2012 was $20.26 \%, 21.45 \%, 25.98 \%, 26.57 \%, 28.68 \%, 29.67 \%$, and $32.63 \%$, respectively. There was an increase in linear trend $\left(\chi 2_{\text {linear trend }}=48.71\right.$, $p<0.001$ ) (Fig. 2).

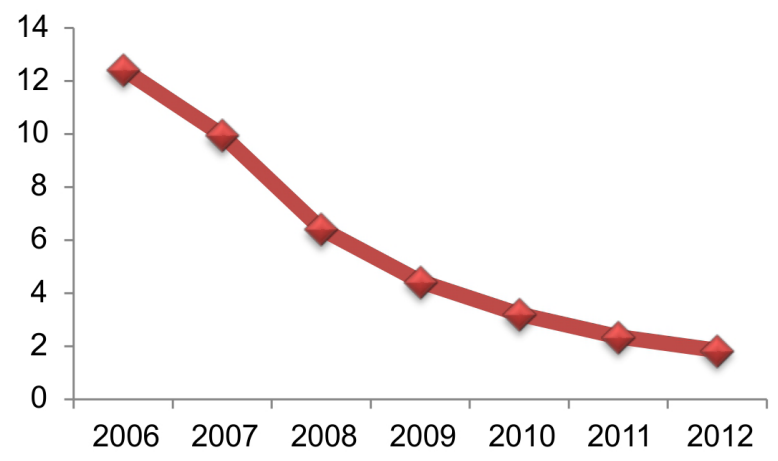

Fig. 1 - Annual occurrence of bacillary dysentery confirmed cases per 100,000 in Guangzhou, southern China, 2006-2012

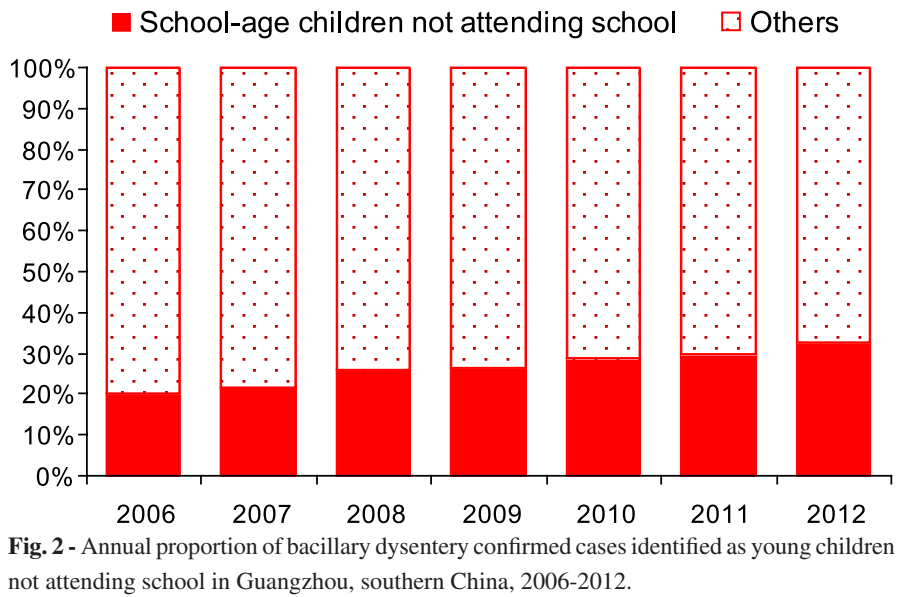

We found in Guangzhou, that the annual incidence rate of BD showed a decreasing trend in the last six years. This result is consistent with the study from some Chinese cities/provinces, such as Henan ${ }^{5}$, Anhui ${ }^{13}$, and Hangzhou $^{7}$ where IIDSPCP was also implemented by the government. However, our results implied that the same or even more attention should 
LI, T.; YANG, Z. \& WANG, M. - Should school-age children not attending school and adults aged 20-44 years be considered high-risk group for bacillary dysentery infection in Guangzhou, China? A review of 4775 bacillary dysentery cases. Rev. Inst. Med. Trop. Sao Paulo, 56(3): 275-6, 2014

be given to school-age children not attending school, since they have become the noticeable infected occupational group in Guangzhou, a trend that appears to have increased over the years. In China, the majority of this group comes from low-income families, their parents usually do not have enough money to send them to Kindergarten or nursery. They are usually lacking in adequate sanitation, with low health risk awareness, and more likely to have exposure to contaminated food or water. It has been reported that compared to the other group, the school-age children not attending school have higher incidence in many infectious diseases such as hand-food-mouth disease ${ }^{9}$, infectious diarrhea ${ }^{14}$, and hepatitis $\mathrm{A}^{4}$. Therefore, more effort should be made to enhance health education and monitoring targeting at school-age children not attending school.

Consistent with the study from $\operatorname{Iran}^{8}$, Egypt $^{1}$, and Chinese other cities $^{11}$, we found the highest proportion of BD cases was in the age group $\leq 5$ years. However, in contrast to earlier findings, current study revealed the second proportion of BD cases was in the age group 20-44 years rather than in age group 6-19 years. One possible explanation for this might be that the vast majority of people aged 6-19 years were school students. Since 2006, as a subprogram of IIDSPCP, preventive strategies have been strengthened in school by increasing or improving the number of hand washing facilities, the frequency of health education course, and the Inspection of food and water hygiene. However, compared to the others, the people aged 20-44 years are more likely to get infected with $\mathrm{BD}$. It is because that people in this age range are in the active stage of social activities; they have more chance to contact with Shigella patients or carriers. Furthermore, these people usually have more leisure time, energy, and finance to have a trip, thus more likely to have exposure to Shigellosis. Although we did not collect information on the proportion of BD cases having travel history, numerous previous studies have revealed that travel was associated with increased risk for shigellosis. For example, in the United States, HALEY et al. interviewed 929 shigellosis cases from Foodborne Diseases Active Surveillance Network, of which 223 (24\%) reported having travelled internationally in the week before symptom onset $^{3}$. In Norway, GUZMAN-HERRADOR et al. found 48.28\% (1435) of gastrointestinal infections were identified as travel-associated during the period of 2009-2010².

Taken together, we reported that with the implement of IIDSPCP, the BD reported cases in Guangzhou demonstrated a decreasing trend in recent years. However, the proportion of cases identified as school-age children not attending school appeared an increasing trend by years and the proportion of adults aged 20-44 years also became higher than before. This information may be useful to establish strategies for prevention, surveillance, and management in China and in other regions or countries where BD typhus is endemic.

Tiegang LI

Zhicong YANG Ming WANG

Correspondence to: Tiegang Li, M.D, Guangzhou Center for Disease Control and Prevention, Guangdong Province, 510440, China. E-mail: tiegang1977@126.com

\section{REFERENCES}

1. El-Gendy AM, Mansour A, Weiner MA, Pimentel G, Armstrong AW, Young SY, et al. Genetic diversity and antibiotic resistance in Shigella dysenteriae and Shigella boydii strains isolated from children aged $<5$ years in Egypt. Epidemiol Infect. 2012;140:299-310

2. Guzman-Herrador B, Vold L, Nygard K. Surveillance of travel-associated gastrointestinal infections in Norway, 2009-2010: are they all actually imported? Euro Surveill. 2012;17:20294

3. Haley CC,Ong KL, Hedberg K, Cieslak PR, Scallan E, Marcus R, et al. Risk factors for sporadic shigellosis, FoodNet 2005. Foodborne Pathog Dis. 2010;7:741-7.

4. Li H, Zhang XS, An J. [Evaluation on the effect of immunization and safety of live attenuated and inactivated hepatitis A vaccine in China]. [Article in Chinese] Zhonghua Liu Xing Bing Xue Za Zhi. 2013;34:24-7.

5. Mu YJ, Zhao JY, Luo Q, Huang LL, Xia SL. Analysis of etiological surveillance results of Shigella spp between 2009 and 2010 in Henan province. [Article in Chinese]. Zhonghua Yu Fang Yi Xue Za Zhi. 2012;46:334-7.

6. Niyogi SK. Shigellosis. J Microbiol. 2005;43:133-43.

7. Pu XY, Pan JC, Wang HQ, Zhang W, Huang ZC, Gu YM. Characterization of fluoroquinolone-resistant Shigella flexneri in Hangzhou area of China. J Antimicrob Chemother. 2009;63:917-20.

8. Shamsizadeh A, Nikfar R, Bavarsadian E. Neurological manifestations of shigellosis in children in southwestern Iran. Pediatr Int. 2012;54:127-30.

9. Sui JL, Wang ZJ. Epidemiological and clinical characteristics on death cases of 635 hand-foot-mouth disease. [Article in Chinese]. Zhonghua Liu Xing Bing Xue Za Zhi. 2012;33:1201-2

10. Sur D, Ramamurthy T, Deen J, Bhattacharya SK. Shigellosis : challenges \& management issues. Indian J Med Res. 2004;120:454-62.

11. Wang SM, Ma JC, Hao ZY, Zhang ZY, Mason C, Sethabutr O, et al. Surveillance of shigellosis by real-time PCR suggests underestimation of shigellosis prevalence by culture-based methods in a population of rural China. J Infect. 2010;61:471-5.

12. World Health Organization. Shigellosis: disease burden, epidemiology and case management. Wkly Epidemiol Rec. 2005;80:94-9.

13. Xiong Z, Li J, Li T, Shen J, Hu F, Wang M. Prevalence of plasmid-mediated quinoloneresistance determinants in Shigella flexneri isolates from Anhui Province, China. J Antibiot (Tokyo). 2010;63:187-9.

14. Ye HC, Liu YH. Epidemiological study of rotavirus diarrhea in Beijing area from 2010 to 2012. [Article in Chinese]. Zhonghua Shi Yan He Lin Chuang Bing Du Xue Za Zhi. 2012;26:432-4.

15. Yuehong Wei, Zhicong Yang, Yan Kang. 2006-2010 Guangzhou bacillary dysentery epidemic trend analysis, 2006-2010. [Article in Chinese]. South China J Prev Med. 2011;37(6):61-3. 